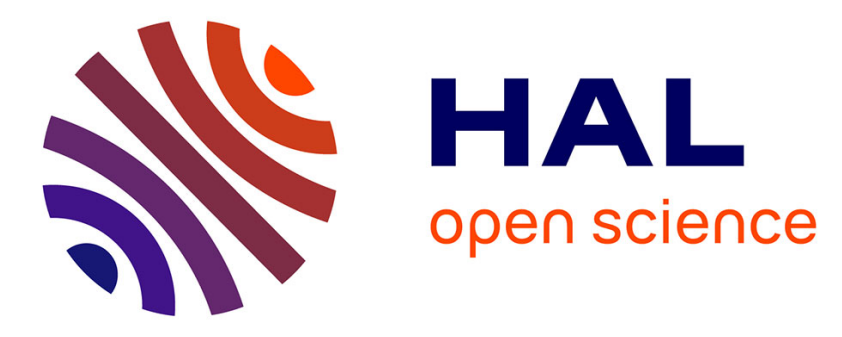

\title{
The magnetic-distortion probe: Velocimetry in conducting fluids
}

Sophie Miralles, Gautier Verhille, Nicolas Plihon, Jean-François Pinton

\section{To cite this version:}

Sophie Miralles, Gautier Verhille, Nicolas Plihon, Jean-François Pinton. The magnetic-distortion probe: Velocimetry in conducting fluids. Review of Scientific Instruments, 2011, 82, pp.095112. 10.1063/1.3630949]. hal-01140480

\section{HAL Id: hal-01140480 \\ https://hal.science/hal-01140480}

Submitted on 9 Apr 2015

HAL is a multi-disciplinary open access archive for the deposit and dissemination of scientific research documents, whether they are published or not. The documents may come from teaching and research institutions in France or abroad, or from public or private research centers.
L'archive ouverte pluridisciplinaire HAL, est destinée au dépôt et à la diffusion de documents scientifiques de niveau recherche, publiés ou non, émanant des établissements d'enseignement et de recherche français ou étrangers, des laboratoires publics ou privés. 


\title{
The magnetic-distortion probe: Velocimetry in conducting fluids
}

\author{
Sophie Miralles, Gautier Verhille, Nicolas Plihon, and Jean-François Pinton \\ Laboratoire de Physique, École Normale Supérieure de Lyon, CNRS UMR 5672 \& Université de Lyon, \\ F-69364 Lyon, France
}

(Received 10 May 2011; accepted 9 August 2011; published online 20 September 2011)

\begin{abstract}
A new type of velocimeter, capable of local velocity measurements in conducting fluids, is introduced. The principle of the "magnetic-distortion probe" is based on the measurement of the induced magnetic field by the flow of a conducting fluid in the vicinity of a localized magnetic field. The new velocimeter has no moving parts, and can be enclosed in a sealed cap, easing the implementation in harsh environments, such as liquid metals. The proposed method allows one to probe both the continuous part and fluctuations of the velocity, the temporal and spatial resolution being linked to the actual geometric configuration of the probe. A prototype probe has been tested in a gallinstan pipe flow and in a fully turbulent flow of liquid gallium generated by the counter rotation of two coaxial impellers in a cylinder. The signals have been compared to a reference potential probe and show very good agreement both for time-averaged velocities and turbulent fluctuations. The prototype is shown to detect motion from a few $\mathrm{cm} \mathrm{s}^{-1}$ to a few $\mathrm{m} \mathrm{s}^{-1}$. Moreover, the use of the magnetic-distortion probe with large-scale applied magnetic field is discussed. () 2011 American Institute of Physics. [doi:10.1063/1.3630949]
\end{abstract}

\section{INTRODUCTION}

Velocity measurement in electrically conducting liquids is an important issue for control and characterization of flows in various situations, ranging from geophysical flows to liquid metals flows in metallurgy. In the industry, metal casting processes require a fine control of the details of the metal flow and, thus, precise and reliable liquid metal flow measurements. In the fission-nuclear industry, cooling of the core could be achieved with flows of liquid $\mathrm{Na}$ or $\mathrm{NaK}$ in fast neutron breeder reactors. Precise and reliable liquid metal flow measurements in the cooling circuitry are thus extremely important for safety issues. On the limit of low-conductivity fluids, some of the food-processing industry processes also rely on the measurements of flows in such liquids. Besides these industrial applications, scientific experiments focussing either on fine characterization of magnetohydrodynamic (MHD) turbulence or on the understanding of the dynamo instability also require precise flow measurements. We propose here a new velocimeter for conducting fluids, with no electrode contact with the fluid, unlike usual velocimeter in conducting fluids. The usual flow measurement techniques, mostly developed to probe velocity fields in water, are usually restricted to media transparent to optical wavelengths. Among these are the Schlieren technique, particule image velocimetry, laser Doppler velocimetry, and particle tracking. Unfortunately these methods cannot be applied to liquid metals which are opaque to optical or near-optical wavelengths. However, a number of flow measurements methods in conducting fluids have been developed over the past decades. These methods can be divided as (integral) flow-rate measurements or (local) velocity probes.

A number of integral flow meters are adapted from non-conducting fluids: momentum-based (such as turbines), pressure-based, or counter-based (measurement of pressure drop or Strouhal number downstream an obstacle). Electro- magnetic flow meters are widely used, either measuring the induced current in the presence of a dc applied magnetic field or measuring the induced magnetic field in the presence of an ac applied magnetic field from a flowing conducting fluid. ${ }^{1}$ Recently, Thess et al. ${ }^{2}$ proposed a method based on the measurement of the back reaction of the flow to a permanent magnet located outside a channel flow; however, the temporal resolution of this method has not been investigated, but might be limited due to inertia.

Local velocity measurement methods are briefly introduced here, but readers are referred to Ref. 3 for a detailed introduction to the field. Non-invasive methods are restricted to ultrasonic methods, ${ }^{4,5} \mathrm{x}$-ray radiography, ${ }^{6}$ or magnetic tomography ${ }^{7}$ in the presence of a large-scale magnetic field. They provide the flow structure without perturbing the fluid, but usually require difficult and costly operation. Invasive probes consist of reaction-force probe, ${ }^{8}$ hot wire probes $^{9}$ (usually restricted to low velocities), or potential difference probes, ${ }^{10}$ which all require either moving parts or electrodes in contact with the fluid. Besides limitations linked to the intrinsic invasiveness of the probes, various practical limitations of these devices are due to temperature, chemical aggressiveness, or electrode pollution (that cannot be easily observed in these opaque fluids).

In this article, we present a new local measurement method based on the interpretation of the magnetic induction from a conducting fluid flow in the presence of a localized magnetic field. Since the velocity is inferred from distortion of a locally applied magnetic field from a flowing electrically conducting fluid, the probe has been named "magnetic-distortion probe." The main asset of the "magnetic distortion probe" is that neither moving parts nor electrical contact with the fluid is necessary, thus easing the assembly, the operation, and the reliability of the probe. The magneticdistortion probe has been calibrated in a gallinstan pipe flow and compared to a reference potential difference probe in a 
fully turbulent gallium flow. The agreement between the measurements of the two probes is excellent both for the continuous part and for low frequencies fluctuations of the velocity components. The principle of the probe is described in details in Sec. II. The gallinstan pipe flow and the turbulent von-Kármán gallium flow are also described in Sec. II. The calibration of the magnetic-distortion probe is discussed in Sec. III, and shows a very good agreement with theoretical calculations. The response of the magnetic-distortion probe in fully turbulent gallium flows is analyzed in Sec. IV both for time-averaged velocities and dynamical response to turbulent fluctuations. Since the method relies on the interpretation of the induction signal, a limitation arises in presence of spurious magnetic field. For instance, parasitic magnetic fields may be induced from gradients of the fluid motion in the presence of a large-scale applied magnetic field. This case is investigated in Sec. V, and a simple correction scheme is shown to accurately cancel the large-scale induced magnetic fields, enhancing the operational range of the probe. Conclusions and perspectives are developed in Sec. VI.

\section{PRINCIPLE OF THE APPARATUS AND EXPERIMENTAL SETUP}

The physical principles underlying the magneticdistortion probe are described in the following subsection II A. Then, the probe design and integration are discussed in Subsection II B. Eventually, the two gallium flows where the probe has been tested and calibrated are presented in Subsection II C for the pipe flow (in Perm, Russia) and in Subsection II D for the turbulent von-Kármán gallium flow (in Lyon, France).

\section{A. Probe principle}

In presence of a time-independent imposed magnetic field $\mathbf{B}_{\mathbf{0}}$, and in the limit of small magnetic Reynolds number regime (e.g., when the induced field $\mathbf{b}$ is small compared to the applied field $\mathbf{B}_{\mathbf{0}}$ ), the induction equation reads

$$
\frac{\partial \mathbf{b}}{\partial t}=\left(\mathbf{B}_{\mathbf{0}} \cdot \nabla\right) \mathbf{u}-(\mathbf{u} . \nabla) \mathbf{B}_{\mathbf{0}}+\lambda \Delta \mathbf{b},
$$

where $\mathbf{u}$ is the conducting fluid velocity and $\lambda=\left(\mu_{0} \sigma\right)^{-1}$ is the magnetic diffusivity with $\mu_{0}$ the magnetic permeability of vacuum and $\sigma$ the electrical conductivity of the fluid. In the remaining of the manuscript, the notation of the magnetic field will be $\mathbf{B}$ for the total magnetic field (i.e., the applied magnetic field and the induced magnetic field) and $\mathbf{b}$ for the induced magnetic field.

The principle of the magnetic-distortion probe ${ }^{11}$ is to measure the source term $(\mathbf{u} . \nabla) \mathbf{B}_{\mathbf{0}}$ in the induction equation, which is proportional to the fluid velocity. The $(\mathbf{u} . \nabla) \mathbf{B}_{\mathbf{0}}$ is the dominant source term of the induction equation when the gradient scale of the imposed magnetic field is smaller than the gradient scale of the velocity field.

The case of a stationary and uniform velocity field in presence of a small magnetic dipole is the simplest case where this assumption holds. The induced magnetic field has been computed in Ref. 12 for this situation. The geometry used in

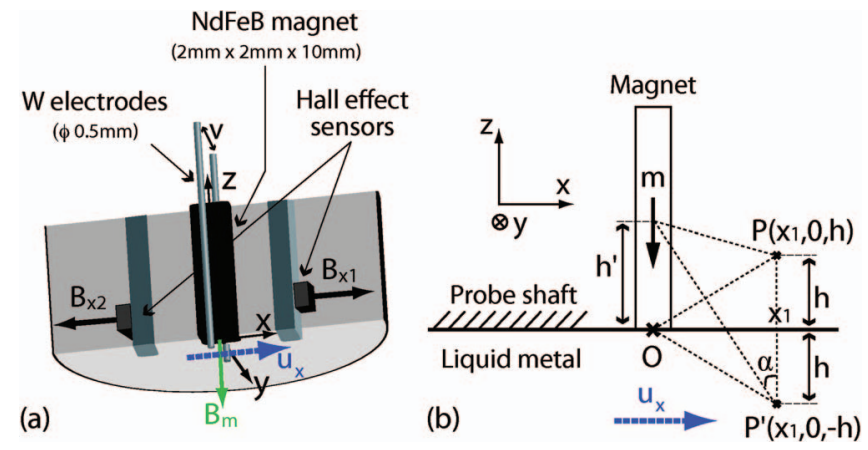

FIG. 1. (Color online) (a) Sketch of the probe assembly showing only two Hall-effect sensors and two tungsten electrodes. The magnetic distortion probe consists of a permanent magnet and magnetic sensors (Hall-effect sensors) probing the induced magnetic field. A reference potential probe is inserted at the same location. (b) Geometry of the magnetic distortion probe used for calculations.

the present manuscript is adapted from Ref. 12 and is recalled in Fig. 1(b). A small permanent magnet, modeled by a dipole $\mathbf{m}$ is set close to a semi-infinite uniform flow of electrically conducting fluid. The material at rest is perfectly insulating. The induced magnetic field at location $P\left(x_{1}, 0, h\right)$ in the presence of a uniform fluid translation with constant velocity $u_{x}$ in half space $z<0$ reads

$$
b_{x}\left(x_{1}, 0, h\right)=-\frac{\mu_{0}^{2} m \sigma u_{x}}{16 \pi x_{1}^{2}} \sin ^{2}\left(\frac{\alpha}{2}\right)\left[4+3 \cos (\alpha)+\cos ^{2}(\alpha)\right],
$$

$$
b_{y}\left(x_{1}, 0, h\right)=0 \text {, }
$$

$$
b_{z}\left(x_{1}, 0, h\right)=-\frac{\mu_{0}^{2} m \sigma u_{x}}{16 \pi x_{1}^{2}} \sin ^{3}(\alpha)
$$

where $\alpha$ is the angle introduced in Fig. 1(b) and defined as $\tan (\alpha)=x_{1} /\left(h+h^{\prime}\right)$. These calculations show that, in presence of a flowing conducting fluid, each component of the induced magnetic field perpendicular to the magnetic dipole is proportional to the velocity component in the same direction. Our magnetic-distortion probe relies on the proportionality of the induced magnetic field with the velocity field. We will show in the following sections that the measurement of a component of the induced magnetic field, close to a small permanent magnet, is indeed a measurement of the local flow velocity. A complete calculation in the presence of a physical boundary layer and with a temporally and spatially fluctuating velocity field is beyond the scope of the present article, but we will show and discuss, from experimental data, the validity of the method.

\section{B. Probe prototype design}

The magnetic-distortion probe prototype consists of a small $\mathrm{NdFeB}$ permanent magnet of size $2 \times 2 \times 10 \mathrm{~mm}$ magnetized along its main axis. The strength of the magnetic field at the magnet surface is $2000 \mathrm{G}(0.2 \mathrm{~T})$, and its direction is sketched in Fig. 1(a) with vector $\mathbf{B}_{\mathbf{m}}$, along the $z$ direction. Four integrated Hall-effect sensors (Micronas HAL 401), 
placed a few $\mathrm{mm}$ apart of the magnetic dipole, probe the magnetic field in the two perpendicular directions to the dipolar applied magnetic field (refer to Fig. 1(a), where only two sensors, probing $\mathbf{B}_{\mathbf{x} 1}$ and $\mathbf{B}_{\mathbf{x} 2}$ are displayed for the sake of clarity). As explained in the previous subsection, the $x$ component of the induced magnetic field is proportional to the $x$ component of the velocity field. With four Hall sensors, the prototype magnetic-distortion probe is a two-axis velocity probe, measuring the $u_{x}$ and the $u_{y}$ velocity components (an extension to a three-axis velocity probe would be straightforward). The signals from the magnetic sensors are fed into AD8221 amplifiers with a gain of 10, and in order to amplify the physical signals and reduce the influence of external parameters (such as temperature) on offset values, our magnetic-distortion probe is built as a pseudo-differential probe: the signals are recorded as the difference between two opposite Hall-effect sensor (i.e., the computation of $\mathbf{b}_{\mathbf{x} 2}-\mathbf{b}_{\mathbf{x} 1}$ enhances the sensitivity to $\mathbf{u}_{\mathbf{x}}$ ). In the case of a perfectly symmetric device, the difference $\mathbf{B}_{\mathbf{x} 2}-\mathbf{B}_{\mathbf{x} 1}$ cancels out the applied field and is equal to $\mathbf{b}_{\mathbf{x} 2}-\mathbf{b}_{\mathbf{x} \mathbf{1}}$ and, thus, directly proportional to the velocity $\mathbf{u}_{\mathbf{x}}$. Due to small asymmetries, ones proceeds to a reference measurement $\mathbf{B}_{\mathbf{x} 2}^{\text {rest }}-\mathbf{B}_{\mathbf{x} 1}^{\text {rest }}$ in the absence of fluid motion, that is always subtracted to the measurements. At the sensor location, the $x$-component of the imposed field is of the order of $20-80 \mathrm{G}$, and the induced component is of the order of a few hundredths to a few gauss in the investigated flows; the noise standard deviation of the Micronas HAL 401 corresponds to $0.02 \mathrm{G}$.

In order to compare the dynamical response of the magnetic-distorsion probe, our prototype design incorporates at the same location a reference potential probe (note that this reference potential probe was only implemented in the von-Kármán gallium flow). Potential probes have widely been used for velocity measurements in different operating conditions $^{3}$ and the characteristics of our design has been described in details in Ref. 13. Four tungsten electrodes (diameter $500 \mu \mathrm{m}$, length $2 \mathrm{~mm}$ ) are set along the magnet sides for the sake of clarity only two electrodes are displayed in Fig. 1(a). The difference of potential $\mathrm{v}$ between these two electrodes (spaced by distance $d \sim 2 \mathrm{~mm}$ ) probes the local velocity field. In Fig. 1(a), the potential difference v probes the electric field in the $y$ direction, which is proportional to the $x$-component of the velocity $\mathbf{u}_{\mathbf{x}}, \mathbf{v}=\int_{0}^{d}\left(\mathbf{u}_{\mathbf{x}} \times \mathbf{B}_{\mathbf{m}}\right) \cdot \mathbf{d l}$.

Our setup, thus, allows a 2-axis reference potential difference measurement of the velocity in the plane perpendicular to the axis of the magnet.

The probe assembly is housed in an electrically grounded stainless steel tube and the surface in contact with the liquid metal was covered by a thin layer of Stycast resin (except the Tungsten electrodes which are in electrical contact with the fluid).

The primary (direct) output voltage between electrodes $\mathrm{v} \sim u_{x} B_{m} d$ is low, of the order of $100 \mu \mathrm{V} / \mathrm{m} \mathrm{s}^{-1}$. It is fed into an AD8221 differential amplifier with a gain of 100, located at the end of the probe housing.

All measurements discussed in the present manuscript were digitized with National Instruments digitizers. In the gallium pipe flow experiment (Perm), the signals were digitized using a NI9239 digitizer at a $2000 \mathrm{~Hz}$ acquisition rate, with

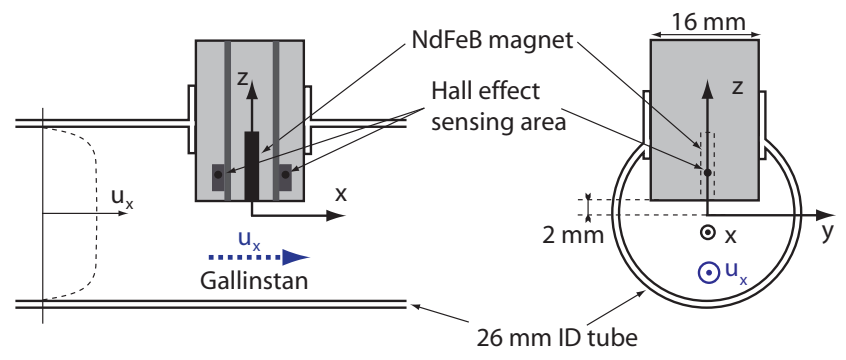

FIG. 2. (Color online) Cross sections of the pipe flow and inserted magneticdistortion probe.

24 bit resolution. In the von-Kármán gallium flow (Lyon), the signals were digitized using a PXI-4472 digitizer with 24 bit resolution at various acquisitions rates (when not specified, the default rate is $1000 \mathrm{~Hz}$ ).

\section{Gallium pipe flow}

The magnetic-distortion probe has been calibrated in a loop pipe flow at the Institute of Continuous Media Mechanics (ICMM), Perm, Russia. The details of the experimental setup can be found in Ref. 14. It consists of a $10 \mathrm{~m}$ long closed pipe flow, where the fluid motion is provided by an inductive 3-phases electromagnetic pump fed with $50 \mathrm{~Hz}$ alternating current. The fluid flow rate can be varied from 0 to $0.21 \mathrm{~s}^{-1}$ either by modifying the current amplitude in the pump or partly closing a valve in the loop. The channel cross section is circular, with inner diameter $2 R_{p}=26 \mathrm{~mm}$. The channel is made of stainless steel and is electrically grounded. The circuit is filled with a gallium alloy (Ga 87.5\%, Sn 10.5\%, $\mathrm{Zn} 2 \%$ ) having the following properties: electrical conductivity $\sigma=3.5610^{6} \mathrm{~S} \mathrm{~m}^{-1}$, density $\rho=6256 \mathrm{~kg} \mathrm{~m}^{-3}$, and kinematic viscosity $v=3.110^{-7} \mathrm{~m}^{2} \mathrm{~s}^{-1}$.

A simplified one axis magnetic-distortion probe has been inserted in the $26 \mathrm{~mm}$ inner diameter channel flow as displayed in Fig. 2. The probe is housed in a $16 \mathrm{~mm}$ diameter grounded stainless steel housing, and measures the streamwise component of the velocity $u_{x}$. In this configuration, the location of the Hall-effect magnetic sensors is $x_{1}=4.7 \mathrm{~mm}$ away from the magnetic axis of the permanent magnet. Since in this setup no precise temperature is available, the use of the pseudo-differential scheme is indeed very useful, accounting for the corrections of the offset variations of the magnetic sensors with the temperature.

A calibrated dc electromagnetic flow meter in the channel flow gives access to the absolute value of the flow rate.

\section{D. von Kármán gallium flow}

Further investigations of the dynamics of the magneticdistortion probe were made in a fully turbulent von-Kármántype gallium flow. This flow is produced by the rotation of two impellers inside a stainless steel cylindrical vessel filled with liquid gallium, as sketched in Fig. 3(a). The cylinder radius $R$ is $97 \mathrm{~mm}$ and its length is $323 \mathrm{~mm}$. The impellers have a radius equal to $82.5 \mathrm{~mm}$ and are fitted to a set of eight blades with height $10 \mathrm{~mm}$. They are separated by a distance $H=203 \mathrm{~mm}$. The impellers are driven by two 


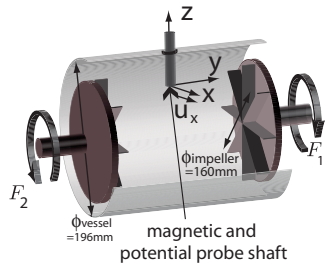

(a) $(\phi=20 \mathrm{~mm})$

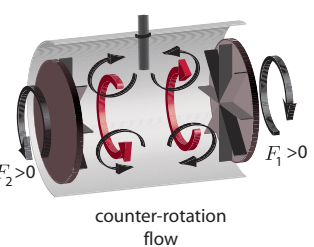

(b)

FIG. 3. (Color online) (a) Sketch of the von-Kármán gallium flow. Schematic structure of the time-averaged flow (b) in the co-rotating regime $\left(F_{2}=-F_{1}\right)$ and (c) in the counter-rotating regime $\left(F_{2}=F_{1}\right)$.

ac-motors which provide a constant rotation rate in the inter$\operatorname{val}\left(\left|F_{1}\right|,\left|F_{2}\right|\right) \in[0.5,25] \mathrm{Hz}$.

The system is cooled by water circulation located behind the driving impellers; the experiments are run at temperatures between $40{ }^{\circ} \mathrm{C}$ and $48{ }^{\circ} \mathrm{C}$ (temperature is kept constant during one run). Liquid gallium has density $\rho=6.09$ $\times 10^{3} \mathrm{~kg} \mathrm{~m}^{-3}$, electrical conductivity $\sigma=3.68 \times 10^{6} \mathrm{~S} \mathrm{~m}^{-1}$, hence a magnetic diffusivity $\lambda=\left(\mu_{0} \sigma\right)^{-1}=0.29 \mathrm{~m}^{2} \mathrm{~s}^{-1}$. Its kinematic viscosity is $v=3.1 \times 10^{-7} \mathrm{~m}^{2} \mathrm{~s}^{-1}$. The integral kinematic and magnetic Reynolds numbers are defined as $\mathrm{Re}=2 \pi R^{2} F / \nu$ and $\mathrm{Rm}=2 \pi R^{2} F / \lambda$. Values of $\mathrm{Rm}$ up to 5 are achieved, with corresponding Re in excess of $10^{6}$.

In the present case, the flow is driven in the "counterrotating" regime $\left(F_{1}=F_{2}=F\right)$. The time-average structure of the flow is sketched in Fig. 3(b): it consists of two cells rotating with opposite toroidal velocities and two recirculation loops close to the impellers, separated by a large shear-layer in the mid plane.

The magnetic-distortion/potential probe is housed in a $20 \mathrm{~mm}$ diameter stainless steel tube and is located in the mid plane between the two impellers (refer to Fig. 3(a)) at a radial location $r=R / 2$. In the sequel, we will restrict the discussion to the characterization of the signals probing the velocity along the azimuthal direction, i.e., the $u_{x}$ component, since the variation of this velocity component with the forcing is the most significant. Note that in this configuration, the Halleffect sensors are located $7 \mathrm{~mm}$ apart from the magnetic axis of the permanent magnet.

\section{CALIBRATION OF THE MAGNETIC-DISTORTION PROBE IN THE PIPE FLOW}

In this section, the theoretical calibration factor relating the value of the $b_{x}$ component of the magnetic field to the $u_{x}$ component of the velocity field in Eq. (2) is compared to an experimental value obtained in the Perm pipe flow. This calibration procedure is then discussed.

The direct time series for both the magnetic-distortion probe and the flow meter are shown in Fig. 4(a) (the signals have been low-pass filtered at $10 \mathrm{~Hz}$, to filter out the $50 \mathrm{~Hz}$ component from the electromagnetic pump). The magnetic distortion probe gives a signal proportional to the flow rate in the pipe. The advantage of using a pseudo-differential scheme can be clearly observed in Fig. 4(b), where the simultaneous time series of the liquid metal temperature and the value of the induced magnetic field from one single sensor (say $\mathbf{b}_{\mathbf{x} \mathbf{1}}$ ) are displayed. When using one single sensor, a clear temper-
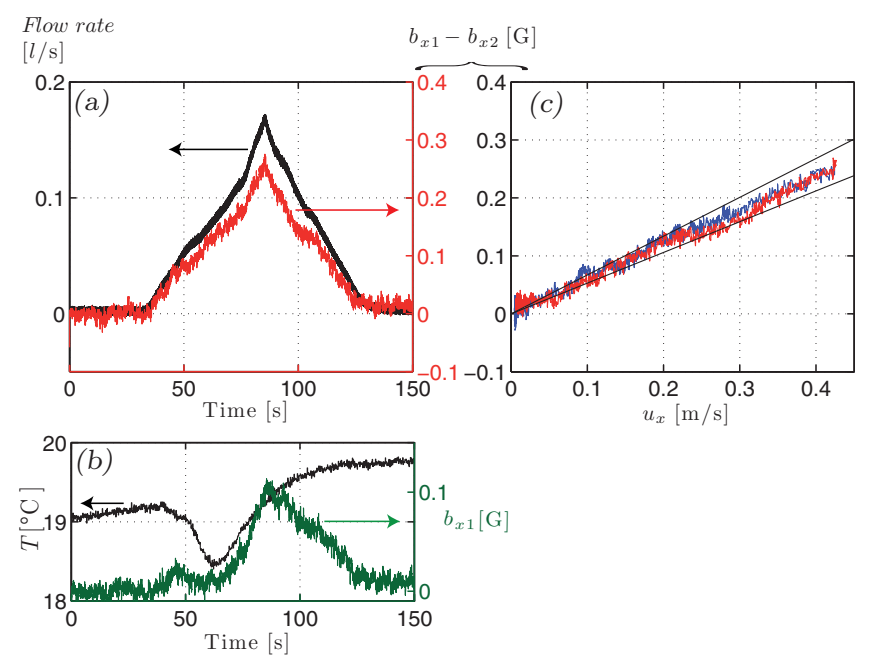

FIG. 4. (Color online) (a) Direct time series of the flow rate from the dc electromagnetic flow meter (upper curve, left ordinate axis) and induced magnetic field (lower curve, right ordinate axis) in the pipe flow for increasing followed by decreasing values of the current in the pump. (b) Direct time series of the fluid temperature (upper curve, left ordinate axis) and induced magnetic field at location $x_{1}$ (lower curve, right ordinate axis) for the same conditions as (a). (c) Evolution of the induced magnetic field as a function of the flow velocity deduced from the dc electromagnetic flow meter (the two curves corresponding to increasing and decreasing values of the flow velocity).

ature variation is observed and cannot be easily corrected for. The pseudo-differential scheme thus provides (i) a direct temperature compensation and (ii) an increase in the sensitivity.

The value of the velocity in the pipe can be calculated from the flow rate $Q$ assuming a (uniform) turbulent profile for the velocity, since the kinetic Reynolds numbers are above 2000 most of the time. However, at the probe location, the cross-section of the flow is $74 \%$ of the circular flow section, so that $u_{x}=Q /\left(0.74 \pi R_{p}^{2}\right)$ (i.e., the maximum streamwise velocity being of the order of $0.4 \mathrm{~m} \mathrm{~s}^{-1}$ ). The evolution of the measured induced magnetic field $\mathbf{b}_{\mathbf{x} 1}-\mathbf{b}_{\mathbf{x} 2}$ as a function of the fluid velocity is displayed in Fig. 4(c). The linearity of the measurement shows that the magnetic-distortion probe indeed measures the fluid velocity.

From these measurements, it is possible to derive an experimental calibration factor $0.6 \pm 0.07 \mathrm{G} / \mathrm{m} \mathrm{s}^{-1}$ (the 0.53 and 0.67 calibration factors are displayed in Fig. 4(c)).

It is possible to compare this experimental calibration factor with a theoretical factor. The calculations done in Ref. 12 assumed the presence of a small magnetic dipole. The magnetic field created by small elongated $\mathrm{NdFeB}$ permanent magnet used in our prototype is compatible with a dipole of amplitude $22 \mathrm{~mA} \mathrm{~m}^{2}$ centered at $h^{\prime}=2.8 \mathrm{~mm}$. With the theoretical geometrical factors of our probe design $\left(x_{1}=4.7 \mathrm{~mm}, h=2.5 \mathrm{~mm}\right.$ ), the calculation gives a theoretical calibration of $1.9 \mathrm{G} / \mathrm{m} \mathrm{s}^{-1}$ (accounting for the fact that the recorded signal is the sum of two induced magnetic fields $\mathbf{b}_{\mathbf{x} \mathbf{1}}$ and $\mathbf{b}_{\mathbf{x} 2}$ ).

Although the theoretical calibration is of the right order of magnitude, the discrepancy with the experimental value is likely to be due to the strong dependence of Eq. (2) on geometrical factors. A calibration procedure is, thus, needed for every magnetic-distortion probe realization, 
since geometrical factors are of leading order. It is important to note here that most of the velocimeters in conducting fluids also require a precise calibration procedure, since they also strongly depend on geometrical factors (electrode location and length for electromagnetic velocimeter; geometry for reaction-based and Lorentz-force velocimetry).

The calibration of the magnetic-distortion probe in a calibrated pipe flow thus showed that:

- the induced magnetic field $\mathbf{b}_{\mathbf{x} 1}-\mathbf{b}_{\mathbf{x} 2}$ is indeed proportional to the fluid velocity $\mathbf{u}_{\mathbf{x}}$;

- the differential scheme increases the sensitivity and is provides a direct temperature compensation;

- the experimental calibration factor is close to the theoretical calibration factor. As geometrical factors have a dominant contribution in the calibration factor, a calibration procedure is required for the magneticdistortion probe, as is for the more usual velocimeters used in conducting fluids.

From these measurements in the pipe flow, the locality of the velocity measurements is not easily determined; an estimate of the flow volume probed by the magnetic-distortion probe is detailed in Sec. IV B.

In the remaining of the text, one will, thus, use the notation "velocity" for the measurements of the magneticdistortion probe.

\section{PROBE CHARACTERIZATION IN FULLY TURBULENT FLOWS}

\section{A. Measurements of flow fluctuations}

In the von-Kármán gallium flow, the typical flow velocity $2 \pi R F$ can reach values of $10 \mathrm{~m} \mathrm{~s}^{-1}$, with high level of fluctuations, providing a test facility for the magneticdistortion probe in ranges unexplored in the pipe flow. However, contrary to the pipe flow, there is no calibrated velocimeter available in the von-Kármán gallium flow; the design of our probe nevertheless incorporates a usual reference potential probe that was shown to accurately probe turbulent fluid velocities. ${ }^{13}$ All results discussed in Secs. IV and V will be non-dimensionalized. In the remaining of the manuscript, the $x$-axis component of the velocity measured by the reference potential probe will be denoted $u_{x}^{\text {pot }}$, while the $x$-axis component of the velocity measured by the magnetic-distortion probe will be denoted $u_{x}^{\text {mag dist }}$. The presence of the probe shaft in the flow vessel indeed modifies the flow characteristics as compared to the ones obtained without probe shaft. However, the modifications of the flow features (mean flow as well as turbulent fluctuations) are similarly probed both by the reference potential probe and the magnetic-distortion probe: our setup is thus suitable for a quantitative comparison of the capabilities of the two velocimeters.

At the probe location, the flow characteristics are the following: the time-averaged velocity is zero, while the turbulent fluctuations standard deviation is of the order of $\pi R F$. The evolution of the standard deviations of the reference potential probe and of the magnetic-distortion probe as a function of the impellers rotation rate is displayed in Fig. 5. This graph

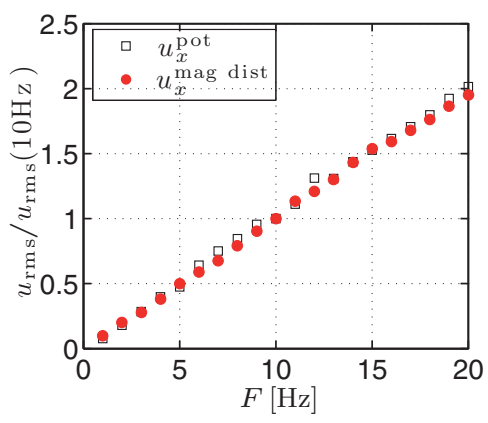

FIG. 5. (Color online) Evolution of the standard deviations of the probed velocities as a function of the forcing frequency $F$ in the von-Kármán flow.

shows the capability of the magnetic-distortion probe to accurately measure fluctuating velocities up to a few $\mathrm{m} \mathrm{s}^{-1}$.

A closer investigation of the dynamics may be gained by analyzing the simultaneous time series of the reference potential probe $u_{x}^{\mathrm{pot}}(t)$ and of the magnetic-distortion probe $u_{x}^{\text {mag dist }}(t)$, as shown in Fig. 6. A clear correlation can be observed between the two signals at low frequencies. The bottom panel of Fig. 6 shows that the magnetic-distortion probe signal filters out fast variations of the velocity field. The correlation between the two signals displayed in Fig. 6 is 0.77 . Note that the value of the correlation between the two signals slightly depends on fine details of the probe geometry (as for the calibration process): values between 0.7 and 0.83 were obtained for different probes with similar operational conditions.

\section{B. Dynamical response}

In this subsection, we will focus on the dynamical aspects of the magnetic-distortion probe.

Typical correlation functions are given in Fig. 7 as a function of the normalized time $t \cdot F$. The correlations are defined as $C_{a b}(\tau)=\int a(t+\tau) b(t) d t$ for signals $a(t)$ and $b(t)$. The correlation-function $C_{u_{x}^{\text {pot }} u_{x}^{\text {mag dist }}}$ is peaked at zero time lag at a value of 0.77 , i.e., the two signals are synchronous and highly
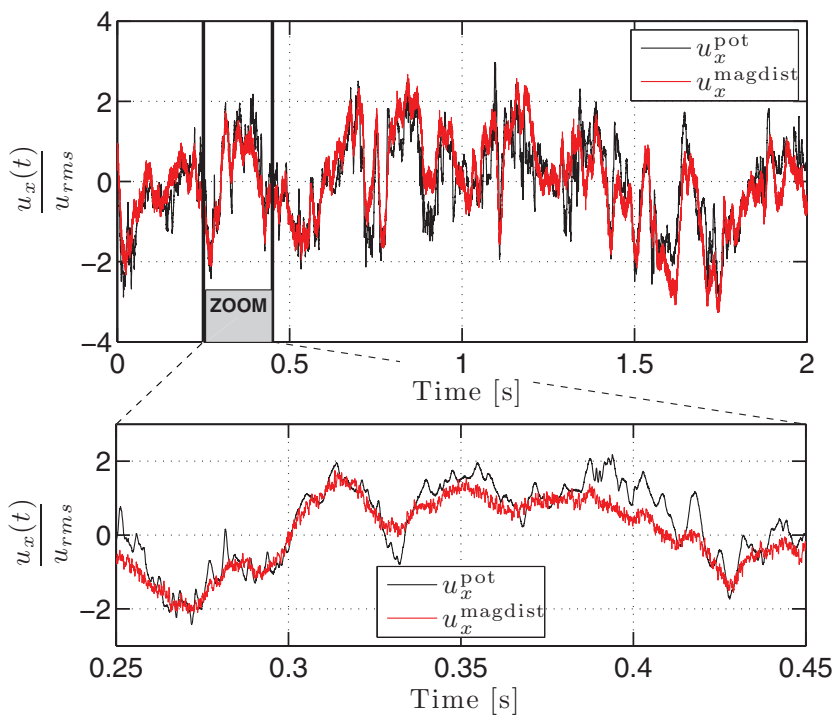

FIG. 6. (Color online) Time signals of reference potential probe and magnetic-distortion probe in the von-Kármán flow in the counter rotating regime $-20 \mathrm{kHz}$ acquisition rate. 


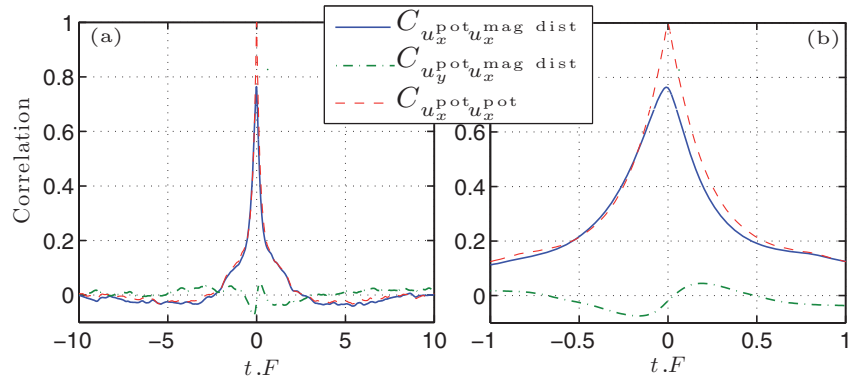

FIG. 7. (Color online) Typical correlation functions in the counter-rotating regime, forcing at $F=10 \mathrm{~Hz}$. See text for details.

correlated. The correlation-function $C_{u_{y}^{\text {pot }} u_{x}^{\text {mag dist }}}$ is nearly zero for all time lags. The magnetic-distortion probe implemented here is thus really a 2 -axis velocity probe.

Let us investigate in details the correlation $C_{u_{x}^{\text {pot }} u_{x}^{\text {mag dist }} \text { and }}$ compare it with the autocorrelation function $C_{u_{x}^{\text {pot }} u_{x}^{\text {pot }} \text {. Both }}$ correlation functions show similar correlation times, with a fast decorrelation time of the order of $T_{i} / 3$ and a slower dynamics with typical time scale $3 T_{i}$, where $T_{i}=1 / F$ is the integral turn-over time. One notes a slight asymmetry of the cross-correlation function (which is also present when considering the signal of only one Hall-effect sensor). When filtering out fluctuations above $3 F$, the asymmetry of the correlation function disappears (not shown). This asymmetry is thus ascribed to induction effects from a turbulent field, as discussed below. Note that there is also a slight increase in the correlation when filtering out high frequency fluctuations (from 0.77 to 0.8 for the signals plotted in Fig. 7).

Details of the dynamics may also be obtained by computing the spectral coherence between the reference potential probe and the magnetic-distortion probe. Figure 8 shows the evolution of the spectral coherence as a function of the frequency $f$, normalized to the impellers rotation rate $F$, for increasing values of the impellers rotation rate $F$ from 4 to $16 \mathrm{~Hz}$. For the highest impeller rotation rates $(F \geq 10 \sim \mathrm{Hz})$, all curves align on a master curve, while for the lowest rotation rates the signal-to-noise ratio decreases and results in a homothetic transformation of the master curve. This curve can be split into three distinct parts:

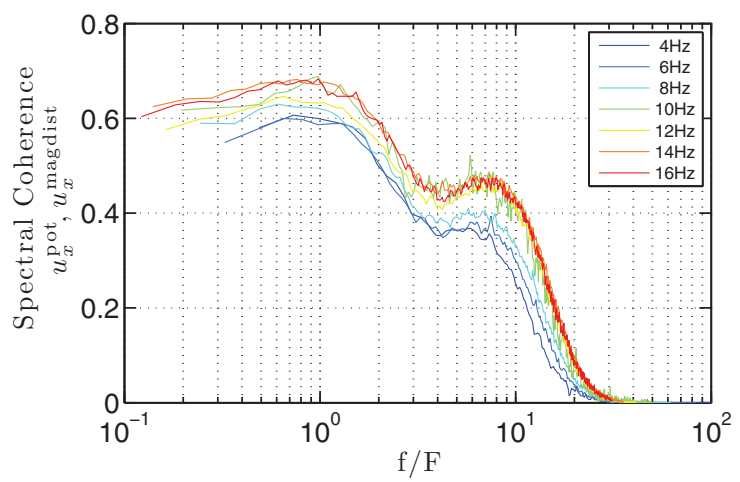

FIG. 8. (Color online) Spectral coherence between the reference potential probe $u_{x}^{\text {pot }}$ and the magnetic-distortion probe $u_{x}^{\text {mag dist }}$ as a function of frequency $f$ normalized to the impellers rotation rate $F$ in the counter-rotating regime. Increasing rotation rate $F$ are referred in the inset.
- At low frequencies $(f<2 F)$, the coherence is nearly constant with values in the range [0.55:0.7]. The value of the correlation coefficient is reminiscent of the values of the spectral coherence at low frequencies, since most of the energy of the signal is present at low frequencies.

- At intermediate frequencies $(2 F<f<10 F)$, the spectral coherence decreases before reaching a plateau around 0.35-0.5.

- At large frequencies $(10 F<f)$, the spectral coherence quickly reaches zero.

These features show that the magnetic-distortion signal probes the dynamics of the fluid velocity up to twice the forcing frequency.

The three regimes described for the spectral coherence master curve can be explained when investigating the power spectral density (PSD) of the signals. Figure 9 displays the normalized PSDs of the reference potential probe (full line) and the magnetic-distortion probe (dashed line). Noise figures were obtained with no motion of the impellers, the probe being inserted in the vessel, filled with liquid gallium. The velocity signals were obtained at a forcing frequency $F$ $=10 \mathrm{~Hz}$. The noise level for both probes has been chosen as the reference $0 \mathrm{~dB}$ level. For the potential probe, this corresponds to the digitizer noise figure. For the magneticdistortion probe, the noise level is set by the noise figure of the magnetic sensor used. As specified in the magnetic sensor HAL 401 application note, a passive low-pass filter was implemented between the sensor and the amplifier, with a cut-off frequency around $2 \mathrm{kHz}$ (as observed in the PSD of Fig. 9). The $0 \mathrm{~dB}$ level of the magnetic-distortion probe was thus chosen as the noise level of the magnetic sensor one decade below the low-pass filter cut-off frequency. The PSDs of the measured velocities are displayed as a solid curve for the reference potential probe $u_{x}^{\text {pot }}$ and as a dashed curve for the magneticdistortion probe $u_{x}^{\text {mag dist }}$.

Let us first investigate the PSD of the reference potential probe. As expected for turbulent hydrodynamic signals, the energy is injected at large scale and cascades towards

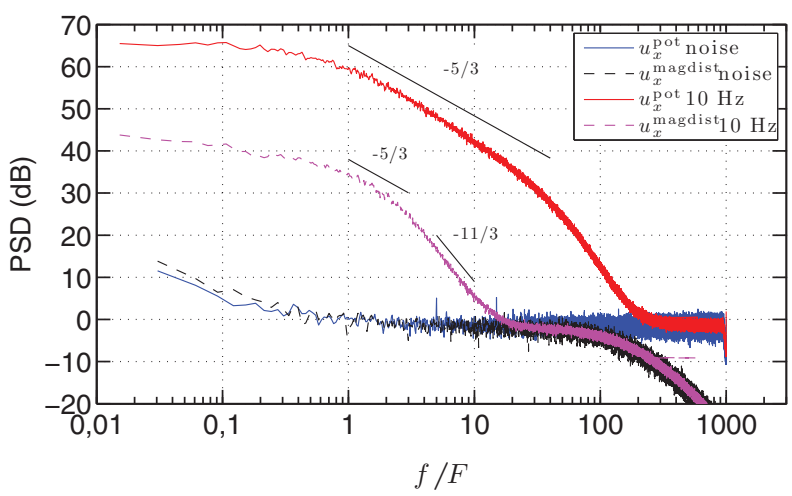

FIG. 9. (Color online) Power spectral densities of noise figure and velocities in the counter rotating case at $F=10 \mathrm{~Hz}$ as a function of the normalized frequency $(f / F)$. Upper solid curve corresponding to $u_{x}^{\text {pot }}$, middle dashed curve corresponding to $u_{x}^{\text {mag dist }}$ in the counter rotating regime, and lower curves correponding to noise figures (see inset). Acquisition rate: $20 \mathrm{kHz}$. 
smaller scales with a $k^{-5 / 3}$ scaling. This turbulent cascade is observed in the time-frequency PSD with a $f^{-5 / 3}$ scaling, observed over 1.5 decade above the injection frequency (here $f / F \sim 1$ ). The finite inter-electrode distance $d$ introduces a physical cut-off frequency of the measurement, which might be estimated from simple arguments on conservation of the turbulent energy $\epsilon=u_{l}^{3} / l \sim f_{l}^{3} l^{2}$ as $f_{c} / F \sim(R / d)^{2 / 3}$. Bolonov et al. ${ }^{15}$ showed that $f_{c} / F=1.5(R / d)^{2 / 3} \approx 20$. The emergence of low frequencies dynamics, typical from flows in such geometries, is observed below the forcing frequency $F$.

Investigation of the PSD of the magnetic-distortion probe also shows the low-frequency dynamics and half a decade of the turbulent cascade (with a $f^{-5 / 3}$ scaling). At frequencies above $3 F$, the spectra shows a $f^{-11 / 3}$ scaling. This scaling is expected for the induced magnetic field from gradients of the turbulent velocity field in a steady-state magnetic field. ${ }^{16}$ As for the potential probe, a similar simple argument for the estimation of the cut-off frequency gives $\left(R / 2 x_{1}\right)^{2 / 3} F \sim 3.3 F$, which is in accordance with the experimental value. The temporal cutoff is thus ascribed to the spatial locality of the velocity measurements by probe. Both from experimental data and Eq. (2), $2 x_{1}$ appears to be the relevant spatial scale. Our prototype probe spatial resolution is thus of the order of $14 \mathrm{~mm}$. We argue here that this scale is closely related to the geometry of the probe and could easily be decreased by modifying the probe design (optimizing the geometry of the locally applied magnetic field and the sensors location).

From these remarks, the shape of the spectral coherence displayed in Fig. 8 can now be better understood. The highly correlated low-frequency part of the spectral coherence $(f \leq 2 F)$ corresponds to the range of velocity measurement by the magnetic-distortion probe. The range of moderate correlation $(4 F<f<10 F)$ corresponds to a range of frequency where the magnetic-distortion probe mostly probes the induced magnetic field from the (local) velocity gradients. Eventually, the high frequencies range $(10 F<f)$ with zero coherence corresponds to a range of frequencies where the induced magnetic field is below the noise resolution of the magnetic sensors. Note that the numerical values separating these parts should only be considered as indicative since they depend on the specific design:

- The cut-off frequency depends on the typical gradient scale of the imposed magnetic field, and the location of the magnetic sensor, i.e., the geometry of our prototype probe.

- The velocimeter dynamics $(50 \mathrm{~dB}$ for our prototype probe) is closely linked to the resolution of the magnetic sensor used (the use of Giant magnetoresistance sensors could increase the resolution).

\section{VALIDITY OF THE METHOD IN PRESENCE OF A LARGE-SCALE INDUCED MAGNETIC FIELD}

The pseudo-differential nature of the magnetic-distortion probe allows a correction from offset variation with operational conditions, but no correction from parasitic magnetic fields. All results shown in Secs. III-IV have been obtained in regimes where no spurious magnetic fields were present. In this section, a method is proposed to extend the domain of validity of the velocimetry method in the presence of spurious magnetic fields. These parasitic magnetic fields could be external magnetic fields (which could easily be subtracted from the probed magnetic field by measuring the perturbing magnetic field not too close to the fluid flow) or induced from gradients of the velocity field (the $\left(\mathbf{B}_{\mathbf{0}} \cdot \nabla\right) \mathbf{u}$ in Eq. (1). This latter case is the most difficult case to correct for, since the statistical and dynamical properties of the large-scale turbulent induced magnetic fields (from velocity gradients to $\left(\mathbf{B}_{\mathbf{0}} \cdot \nabla\right) \mathbf{u}$ term) are similar to those of the induced magnetic field from velocity field $\left((\mathbf{u} \cdot \nabla) \mathbf{B}_{\mathbf{0}}\right.$ term $)$. We show that it is possible to correct the measurement from the existence of a large-scale magnetic field (both a steady-state magnetic field and a purely fluctuating magnetic field).

In the presence of a large-scale, steady-state, and homogeneous magnetic field $\mathbf{B}_{\mathbf{0}}^{\mathbf{L}}$ and close to the permanent magnet $\mathbf{B}_{\mathbf{0}}^{\mathbf{M}}$, the source terms of the induction Eq. (1) can be divided into two contributions:

- one term proportional to the large-scale magnetic field and the gradients in velocity: the $\left(\mathbf{B}_{\mathbf{0}}^{\mathbf{L}} \cdot \nabla\right) \mathbf{u}$ term;

- two terms proportional to the localized magnetic field $\mathbf{B}_{\mathbf{0}}^{\mathbf{M}}$ : the $\left(\mathbf{B}_{\mathbf{0}}^{\mathbf{M}} \cdot \nabla\right) \mathbf{u}$ term and the $(\mathbf{u} \cdot \nabla) \mathbf{B}_{\mathbf{0}}^{\mathbf{M}}$ term . With restrictions given in Sec. IV (i.e., for moderate frequencies), the $(\mathbf{u} \cdot \nabla) \mathbf{B}_{\mathbf{0}}^{\mathbf{M}}$ term dominates and accurately probes the fluid velocity.

Far away from the permanent magnet, only the first term $\left(\left(\mathbf{B}_{\mathbf{0}}^{\mathbf{L}} \cdot \nabla\right) \mathbf{u}\right)$ obviously remains.

In Secs. III-IV, the velocity field was probed with a pseudo-differential scheme. Analysis of the data showed that all results remained unchanged when probing the induced magnetic field only at one location (for instance $x_{1}$ in Fig. 1), if all operating conditions can be kept constant. Using this property, we built a modified version of the magneticdistortion probe allowing for the correction of large-scale magnetic field contributions, as displayed in the bottom panel of Fig. 10. The difference from the version shown in Fig. 1 lies in the location of the Hall sensors. One sensor probes the $x$ component of the induced magnetic field at location $\left(x_{1}, 0, z_{1}\right)$ (which is the same location as in Fig. 1), and the other sensor probes the $x$-component of the induced magnetic field at location $\left(-x_{1}, 0, z_{2}\right)$ with $z_{2}=13 \mathrm{~mm}>z_{1}=3 \mathrm{~mm}$. We will focus our attention on a situation with a large-scale magnetic field $\mathbf{B}_{\mathbf{0}}^{\mathbf{L}}$ applied from coaxial coils along the axis of the von-Kármán flow as depicted in Fig. 10. The source terms in the induction equation along the $x$-component are composed:

- (at location $z_{1}$ ) of contributions both from the largescale magnetic field and from the localized magnetic field: $\left(u_{x} \cdot \nabla\right) B_{0}^{M}+\left(B_{0}^{L} \cdot \nabla\right) u_{x}$

- (at location $z_{2}$ ) of a single contribution from the largescale magnetic field: $\left(B_{0}^{L} \cdot \nabla\right) u_{x}$.

The correction scheme is the following: the induced magnetic field at location $z_{2}$ is subtracted from the induced magnetic field at location $z_{1}$ to correct for the large-scale magnetic field contribution. In the counter-rotating regime, the large 
shear layer provides a very efficient large-scale source term $B_{0}^{L} \partial u_{x} / \partial y$, which is usually referred as the $\Omega$-effect in dynamo theory. The features of this induction effect have been widely studied in turbulent von-Kármán flows: ${ }^{16-18}$ a timeaveraged induced magnetic field is efficiently generated by the differential rotation, with turbulent fluctuations intensity of the order of the time-averaged intensity. Along a radius, the correlation of the induced magnetic field is large (of the order of 0.98 for two locations $1 \mathrm{~cm}$ apart). These properties, thus, allow us to consider $b_{x}^{I}\left(z_{2}\right)$ as a correct estimate of the induced magnetic from the $\left(B_{0}^{L} \cdot \nabla\right) u_{x}$ term at location $z_{1}$.

As in Secs. III-IV, a reference potential probe is inserted in the setup, for comparison and validation of the correction scheme. In the sequel, we will refer to the induced magnetic field $b_{x}^{I}\left(z_{1}\right)$ as the uncorrected magnetic-distortion probe signal $u_{x}^{\text {uncorr mag dist; }}$; we will refer to $b_{x}^{I}\left(z_{1}\right)-b_{x}^{I}\left(z_{2}\right)$ as the corrected magnetic-distortion probe signal $u_{x}^{\text {corr mag dist }}$.

Let us first investigate the standard deviations of the signals when increasing the intensity of the large-scale applied magnetic field $B_{0}^{L}$ for a constant flow forcing, as shown in Fig. 11(a). The reference potential probe is not influenced by the presence of such a moderate large-scale applied magnetic field, as shown by the squares. The uncorrected magnetic-distortion probe signal (displayed as stars) increases with $B_{0}^{L}$. This increase is associated with the $B_{0}^{L} \partial u_{x} / \partial y$ induction term, which is linear in $B_{0}^{L}$ and appears not to be correlated with the $\left(u_{x} \cdot \nabla\right) B_{0}^{M}$ term, since the increase is observed for low values of $B_{0}^{L}$. The standard deviation of the corrected magnetic-distortion probe signal is displayed as dots in Fig. 11(a) and is kept constant for moderate intensities of the applied magnetic field $B_{0}^{L}$. The correction scheme appears to be valid up to $B_{0}^{L}=20 \mathrm{G}$, which is remarkably high since, for this applied magnetic field intensity, the standard deviation of the large-scale induced magnetic field is three times larger than the locally induced field.

The correction scheme might also be quantified by the correlation between the reference potential probe and the magnetic-distortion probe. Figure 11(b) shows that the correlation for the uncorrected magnetic-distortion probe (stars) quickly decreases as the large-scale magnetic field amplitude $B_{0}^{L}$ increases. Since the large-scale induced magnetic field contribution is dominant, even for moderate values of the large-scale magnetic field, this feature is expected since turbulent large-scale induced magnetic fields are not correlated with local turbulent velocity fields. The correlation for the corrected magnetic-distortion probe is displayed in the same graph, as dots. A large increase of the correlation is observed when applying the correction scheme, which also appears to be valid up to applied field of the order of $20 \mathrm{G}$. The correction is obviously not very accurate for vanishing values of the applied magnetic field $B_{0}^{L}$ : the assumption that, for the induced field at location $z_{2}$, there is no contribution from the localized magnetic field (permanent magnet) is, strictly speaking, not valid. The amplitude of the induced field $B_{x}^{I}\left(z_{2}\right)$ with $B_{0}^{L}=0$ is indeed around $20 \%$ of the amplitude of $B_{x}^{I}\left(z_{1}\right)$. The simple implementation of the correction scheme presented here could of course be improved by designing more carefully the probe

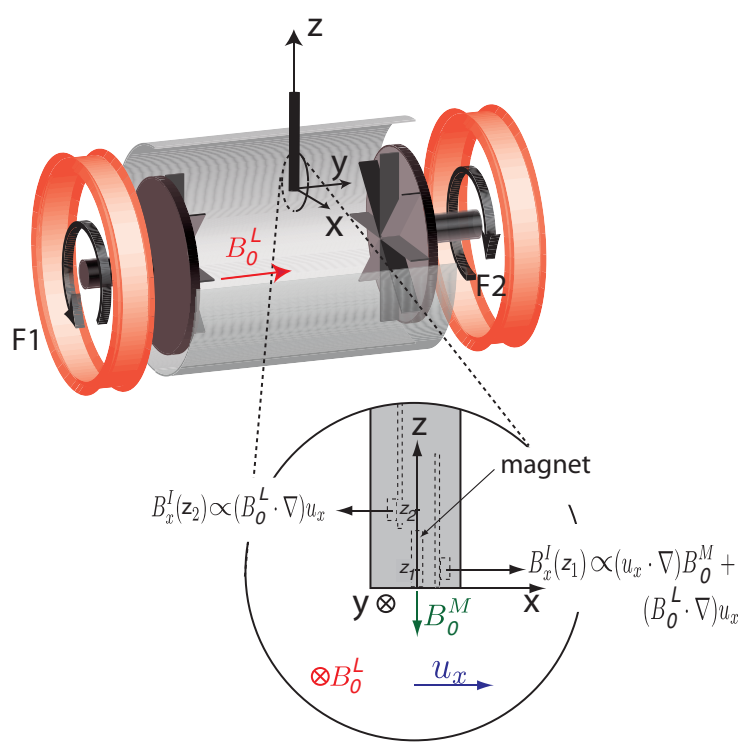

FIG. 10. (Color online) Scheme of the experimental and the magnetic distortion probe in the presence of a large-scale applied magnetic field $\mathbf{B}_{\mathbf{0}}^{\mathbf{L}}$ from coaxial coils in the von-Kármán flow. For clarity, the electrode of the potential probe is not displayed.

(localized magnetic field configuration, location of magnetic sensors).

The validity of the correction scheme can also be investigated in the time-frequency domain. Figure 12 displays the PSD with and without the correction scheme. Once again, the influence of moderate large-scale applied magnetic field intensities is shown to be negligible on the reference potential probe: no change in the PSD of the potential probe with $B_{0}^{L}=0$ (upper dashed curve) or $B_{0}^{L}=15 \mathrm{G}$ (upper solid curve) is observed. On the contrary, the PSD of the uncorrected magnetic-distortion probe at $B_{0}^{L}=0$ (lower dashed curve) and $B_{0}^{L}=15 \mathrm{G}$ (middle solid curve) are strongly different, especially at low frequencies, in a frequency domain that was shown to be the most accurate for the magnetic-distortion probe velocity measurement. The corrected magnetic-distortion probe PSD (displayed as a lower solid curve) is very close to the $B_{0}^{L}=0$ case, which shows
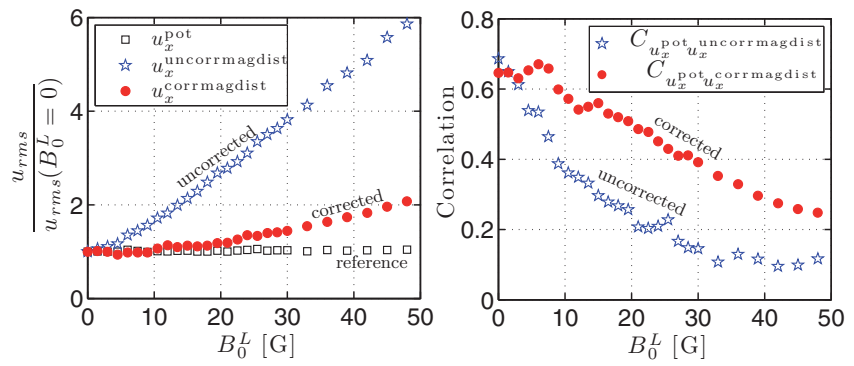

FIG. 11. (Color online) (a) Evolution of the standard deviation as a function of the intensity of the large-scale applied magnetic field $B_{0}^{L}$ for the uncorrected magnetic distortion probe $u_{x}^{\text {uncorr mag dist }}$ (stars), corrected magnetic distortion probe $u_{x}^{\text {corr mag dist }}$ (dots), and reference potential probe $u_{x}^{\text {pot }}$ (squares). (b) Evolution of the correlation coefficient between $u_{x}^{\text {pot }}$ and $u_{x}^{\text {uncorr mag dist }}$ (stars) and between $u_{x}^{\text {pot }}$ and $u_{x}^{\text {corr mag dist }}$ (dots) as a function of the intensity of the large-scale applied magnetic field $B_{0}^{L}$. Counter-rotating flow at $F=10 \mathrm{~Hz}$. 


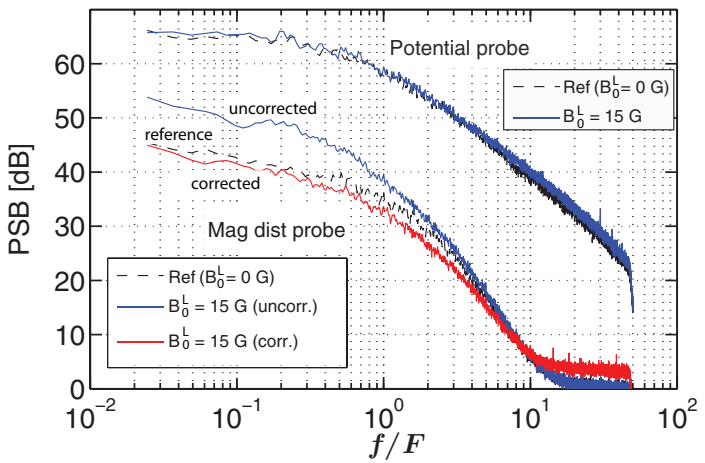

FIG. 12. (Color online) Power spectral density of the reference potential probe with no large-scale applied magnetic field (upper dashed curve) and with $B_{0}^{L}=15 \mathrm{G}$ (upper solid curve). Associated power spectral densities of the magnetic distortion probe with no large-scale applied magnetic field (lower dashed curve) and with $B_{0}^{L}=15 \mathrm{G}$ for the uncorrected magneticdistortion probe (middle solid curve) and corrected magnetic-distortion probe (lower solid curve). Counter-rotating flow at $F=10 \mathrm{~Hz}$.

that the correction scheme indeed extends the domain of validity of the probe in the presence of large-scale applied magnetic fields.

The simple correction scheme introduced in this section is shown to accurately probe turbulent velocity fields in the presence of large-scale applied and large-scale turbulent induced magnetic field. The correction is remarkably efficient, even with the extremely simple implementation, since noisy turbulent induced magnetic fields three-times larger than the useful signal are corrected for. As was pointed out in Secs. III-IV, the experimental data presented here were obtained with a simple prototype probe and the results could be enhanced with an optimized probe design.

\section{CONCLUSION}

A new local velocimeter for electrically conducting fluids was introduced. The fluid velocity is computed from the induced magnetic fluid due to the interaction of the moving electrically conducting fluid with a localized applied magnetic field. Since the fluid velocity is inferred from the distortion of a localized magnetic field, the velocimeter was named "magnetic-distortion probe." Based on previous calculations, a simple prototype probe was built around an elongated permanent magnet, with integrated Hall-effect sensors as magnetic sensors for the induced magnetic field. The ability of the magnetic-distortion probe to measure fluid velocity from a few $\mathrm{cm} \mathrm{s}^{-1}$ to a few $\mathrm{m} \mathrm{s}^{-1}$ was shown in liquid gallium flows. To summarize, the experimental tests of the prototype magnetic-distortion probe thus showed:

- the proposed method allows to probe laminar as well as turbulent fluid flow velocities in liquid gallium flows;

- the probe is a local probe; the locality of the measurement is closely linked to the geometry of the probe, namely, the largest of (i) the scale of the permanent magnetic field gradient or (ii) the location of the magnetic sensor. In our simple prototype, it was shown that the flow is averaged over $14 \mathrm{~mm}$;
- the dynamics of our simple prototype is $50 \mathrm{~dB}$ for velocities in the $\mathrm{m} \mathrm{s}^{-1}$ range;

- fluctuations up to a few tens of Hertz were easily probed;

- a simple correction method was implemented and shown to increase the domain of validity, even in the presence of parasite magnetic fields (even when the statistical properties of these parasite magnetic fields are similar to the velocity signal);

- no moving part nor electrical contact with the electrically conducting fluid are required, easing implementation, sealing, and chemical compatibility in harsh environments.

We argue that the probe characteristics could easily be increased by optimizing the probe geometry and magnetic field sensors. Our prototype probe shares several features with the dynamics of potential probe. However, the implementation and use of the magnetic-distortion probe has several advantages as compared to the potential probes: absence of moving parts, no sealing at the electrode location, no concern of chemical compatibility nor wetting issues between the fluid and the electrodes, no electrode contamination (either chemical or physical), and ease of operation in electrically noisy environments.

Our prototype results open a wide area of applications. For instance, this velocimeter could be used in dynamo experiments to probe how the velocity field is modified when the dynamo magnetic field saturates. An attempt to such measurements was made in the Karlsruhe dynamo experiment ${ }^{19}$ with potential probe. The use of the magnetic-distortion probe in the von-Kármán Sodium experiment ${ }^{20}$ could provide useful physical informations.

\section{ACKNOWLEDGMENTS}

We acknowledge the technical support of M. Tanase and M. Moulin. This work has benefited from discussions with R. Volk. The ICMM team in Perm (R. Halilov, I. Kolesnichenko, S. Kripchenko, and P. Frick) is acknowledged for their welcome and the use of data obtained in the pipe flow. This work was supported by the Contract ANR-08-BLAN0039-02 and CNRS-RFBR Grant No. 07-01-92160.

${ }^{1}$ T. Schulenberg and R. Stieglitz, Nucl. Eng. Des. 240, 2077 (2010).

${ }^{2}$ A. Thess, E. V. Votyakov, and Y. Kolesnikov, Phys. Rev. Lett. 96, 164501 (2006).

${ }^{3}$ S. Eckert, A. Cramer, and G. Gerbeth, Magnetohydrodynamics, Fluid Mechanics and Its Applications 80, 275 (2007)

${ }^{4}$ Y. Takeda, Nucl. Technol. 79, 120 (1987).

${ }^{5}$ D. Brito, H. C. Nataf, P. Cardin, J. Aubert, and J. P. Masson, Exp. Fluids 31, 653 (2001).

${ }^{6}$ Y. Saito, K. Mishima, Y. Tobita, T. Suzuki, and M. Matsubayashi, Exp. Therm. Fluid Sci. 29, 323 (2005).

${ }^{7}$ F. Stefani and G. Gerbeth, Inverse Probl. 15, 771 (1999).

${ }^{8}$ S. Eckert, W. Witke, and G. Gerbeth, Flow Meas. Instrum. 11, 71 (2000).

${ }^{9}$ M. Sajben, Rev. Sci. Instrum. 36, 945 (1965).

${ }^{10}$ R. Ricou and C. Vives, Int. J. Heat Mass Transfer 25, 1579 (1982).

${ }^{11}$ J. F. Pinton, N. Plihon, G. Verhille, and S. Miralles, patent FR1054250, (June 1, 2010).

${ }^{12}$ A. Thess, E. Votyakov, B. Knaepen, and O. Zikanov, New J. Phys. 9, 299 (2007). 
${ }^{13}$ V. Noskov, R. Stepanov, S. Denisov, P. Frick, G. Verhille, N. Plihon, and J. F. Pinton, Phys. Fluids 21, 045108 (2009).

${ }^{14}$ I. Kolesnichenko and P. Frick, Magnetohydrodynamics 45, 165 (2009).

${ }^{15}$ N. I. Bolonov, A. M. Kharenko, and A. E. Eidel'man, J. Eng. Phys. Thermophys. 31(2), 904 (1976).

${ }^{16}$ P. Odier, J. F. Pinton, and S. Fauve, Phys. Rev. E 58, 7397 (1998).

${ }^{17}$ R. Volk, P. Odier, and J. F. Pinton, Phys. Fluids 18, 085105 (2006).
${ }^{18}$ G. Verhille, N. Plihon, M. Bourgoin, P. Odier, and J. F. Pinton, Space Sci. Rev. 152, 543 (2010).

${ }^{19}$ U. Muller, R. Stieglitz, and S. Horanyi, J. Fluid Mech. 552, 419 (2006).

${ }^{20}$ R. Monchaux, M. Berhanu, S. Aumaître, A. Chiffaudel, F. Daviaud, B. Dubrulle, F. Ravelet, S. Fauve, N. Mordant, F. Pétrélis, M. Bourgoin, P. Odier, J.-F. Pinton, N. Plihon, and R. Volk, Phys. Fluids 21, 035108 (2009). 